\title{
Análise espectral do sinal geofísico: exemplos de aplicação da transformada de ondeleta
}

\author{
Júlia Clícia Carvalho de Andrade Amorim, Luciana Figueiredo Prado e Elder Yokoyama
}

Copyright 2019, SBGf - Sociedade Brasileira de Geofísica

This paper was prepared for presentation during the $16^{\text {th }}$ International Congress of the Brazilian Geophysical Society held in Rio de Janeiro, Brazil, 19-22 August 2019.

Contents of this paper were reviewed by the Technical Committee of the $16^{\text {th }}$ International Congress of the Brazilian Geophysical Society and do not necessarily represent any position of the SBGf, its officers or members. Electronic reproduction or storage of any part of this paper for commercial purposes without the written consent of the Brazilian Geophysical Society is prohibited.

\begin{abstract}
The study of natural signals demands robust techniquest to allow comprehension of active physical mechanisms. However, many of these signals show non-stationary characteristics, whose average rate varies over time, preventing the applications of classic spectral methods, such as the periodogram. The wavelet transform is a robust tool for non-stationary time series analysis and its use has spread in this field due to its ease of application through subroutines in several softwares. In this work, we present wavelet transform applied to two series of natural signals: number of sunspots and precipitation in Brasilia, Central Brazil. The goal was to identify cycles in both series and relate them to phenomena described in the literature, through the wavelet transform. Results showed a spectral peak at 11 years in Sunspot Series, related to Schwabe's cycle. For the Precipitation Series, a primary spectral peak of 21 years was identified, probably related to the temperature variance in the Pacific Ocean, and secondary cyclicity in 10-16 months. These results confirm the wavelet transform as a robust and satisfactory tool in the spectral study of non-stationary series.
\end{abstract}

\section{Introdução}

O Sol é a fonte basal de energia na Terra e também sua principal forçante climática externa. Dada essa premissa, como a atividade solar modula a quantidade de energia solar recebida pela Terra, processos climáticos podem ser afetados pela variabilidade solar. A variabilidade solar ocorre em diversas escalas temporais, de segundos a milhares de anos. Em escala anual, observa-se variações no número de manchas solares na fotosfera solar associadas com a quantidade de radiação emitida pelo Sol (Echer et al, 2003). A ocorrência e quantidade das manchas é devida à variação na intensidade do campo magnético poloidal e toroidal solar, cujas linhas de campo interagem e geram protuberâncias na superfície solar. Esse processo ocorre aproximadamente a cada 11 anos e é denominado ciclo de Schwabe (Gómez et al, 2018).

Identificar a atuação de processos cíclicos externos na variabilidade climática pode ser útil para compreender processos climáticos, bem como para melhorar sua previsibilidade. Contudo, muitos sinais geofísicos, como a exemplo da emissão de radiação eletromagnética solar, apresentam característica não-estacionária, cuja média varia ao longo do tempo, e não permite a aplicação de métodos espectrais clássicos, como o periodograma. Nesse contexto, a transformada de ondeleta é uma ferramenta robusta para análise espectral de sinais nãoestacionários. A transformada de ondeleta consiste de funções geradas por dilatações e translações através de uma ondeleta base (Bolzan, 2004).

No caso de sinais geofísicos, a função ondeleta base mais utilizada é a do tipo Morlet (Figura 1), pois esta possui formato similar aos sinais naturais e têm se mostrado útil na identificação das diversas frequências encontradas no sistema natural (Bolzan, 2004).

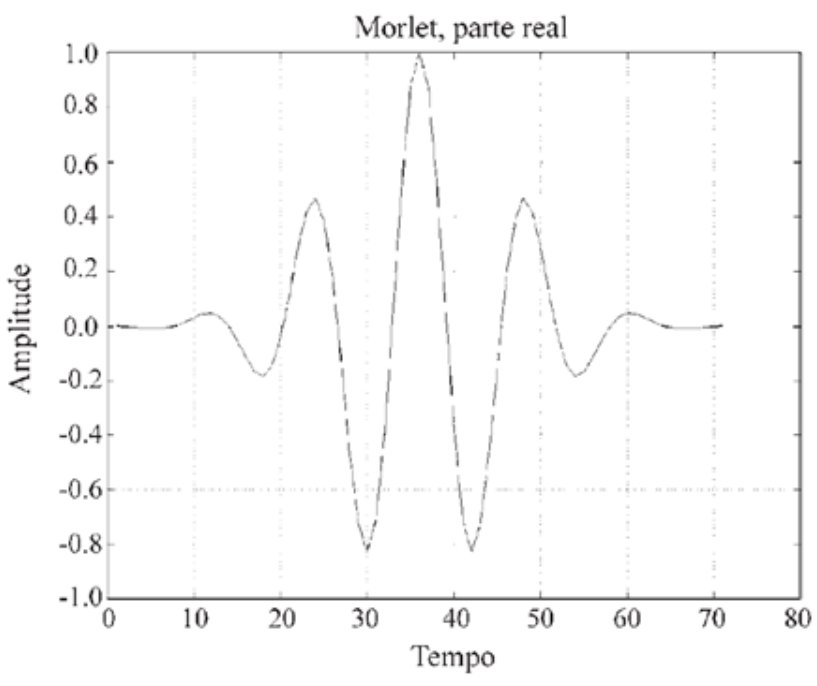

Figura 1. Função ondeleta de Morlet. Adaptado de Bolzan, 2004.

Nesse cenário, o presente trabalho teve como objetivo testar a aplicação do método da transformada de ondeletas como ferramenta estatística na análise de sinais geofísicos não-estacionários, dados por séries de variabilidade climática e solar. A variabilidade climática estudada corresponde à série mensal de precipitação em Brasília, no Brasil Central, enquanto que a variabilidade solar corresponde à série de número de manchas solares, ambas no período de 1962 a 2016. Os resultados aqui apresentados são preliminares. Como etapas futuras, pretende-se investigar possíveis relações 
entre a variabilidade climática no Brasil Central e a variabilidade solar.

\section{Método}

Foram utilizadas duas séries temporais com resolução mensal: a série de precipitação no período de janeiro/1962 a dezembro/2016, provenientes da estação meteorológica de Brasília (latitude -15,78 longitude 47,93; altitude $1159,54 \mathrm{~m}$ ) (Figura 2), do Instituto Nacional de Meteorologia (INMET) - os dados estão disponíveis para download no portal do INMET (http://www.inmet.gov.br/); e a série de manchas solares do Sunspot Index and Long Term Solar Observations (SILSO) - os dados estão disponíveis para download no portal do SILSO (http://sidc.be/silso/home).

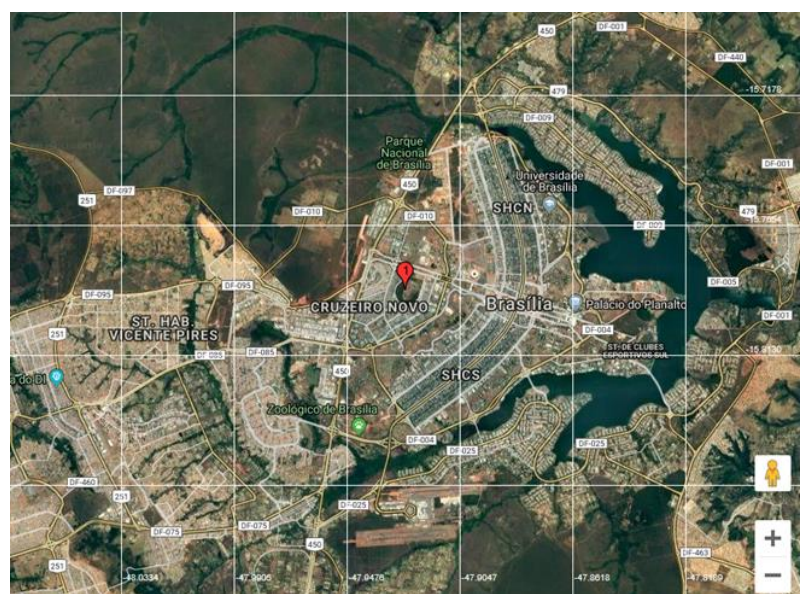

Figura 2. Mapa de Brasília-DF, onde a estação meteorológica de Brasília está marcada pelo balão vermelho (latitude $-15,78^{\circ}$; longitude $-47,93^{\circ}$; altitude 1159,54 m). Fonte: https://earthexplorer.usgs.gov/.

A partir da série de precipitação da estação meteorológica Brasília foi possível calcular o ciclo anual e obter a série de anomalias mensais, que foi normalizada pelo respectivo desvio padrão. Em seguida, para investigar possíveis periodicidades, aplicou-se a transformada de ondeletas tanto na série de anomalias de precipitação quanto na série de manchas solares, utilizando a ondeleta Morlet (Figura 1). Todos os cálculos foram feitos utilizando o software livre R Statistics.

\section{Resultados}

A Figura 3 apresenta as séries temporais mensais de número de manchas solares (Figura $3 \mathrm{~A}$ ), e a série de anomalias de precipitação (Figura $3 \mathrm{~B}$ ), no período de 1965 a 2016. O número de manchas solares (Figura 3A) varia ao longo do tempo, onde se observa a ocorrência de máximos e mínimos, além de um ciclo bem definido. Ao longo do intervalo temporal de análise (1965-2016) são observados 5 ciclos completos. A série normalizada de anomalias de precipitação na estação Brasília (Figura 3B) apresenta variação em magnitude ao longo do tempo. Eventos extremos de chuva acima da média (> +3) apresentam maior magnitude do que eventos extremos abaixo da média $(<-3)$.
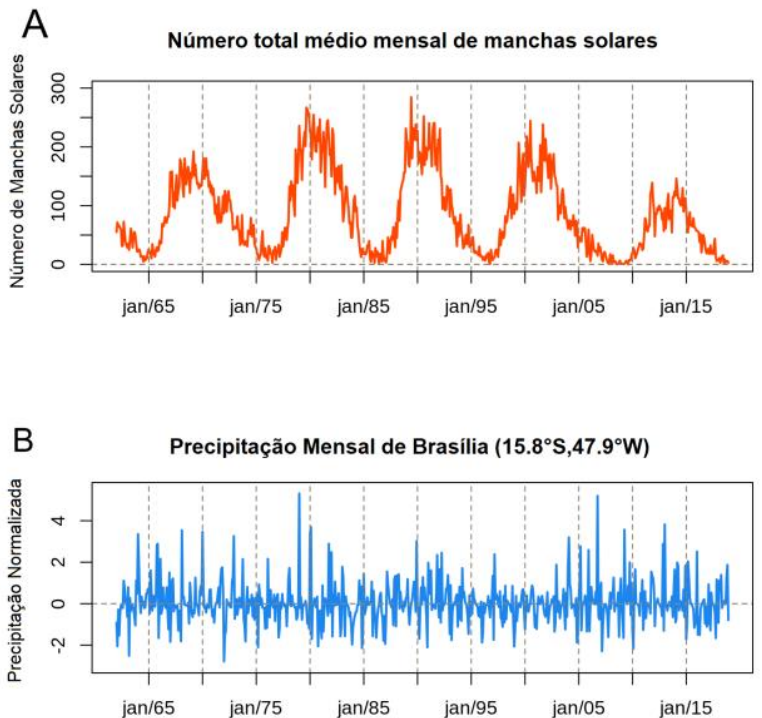

Figura 3. Gráficos de variação de precipitação e manchas solares em relação ao tempo. (A) Série de número mensal de manchas solares, sendo o eixo $Y o$ número de manchas solares e em $X$ o tempo (em anos); (B) Série de anomalias mensais normalizadas de precipitação, sendo o eixo Y a anomalia de precipitação normalizada e em $X$ o tempo (em anos).

O espectro de ondeletas da série de número de manchas solares (Figura 4A) mostra períodos com maior potência em 128 meses (Figura 4B), ou seja, 11 anos, ao longo de todo o intervalo de tempo, com $95 \%$ de significância estatística. Períodos de alta frequência também foram identificados em aproximadamente 64 meses (5 anos), e baixa frequência (260 meses, 22 anos). 

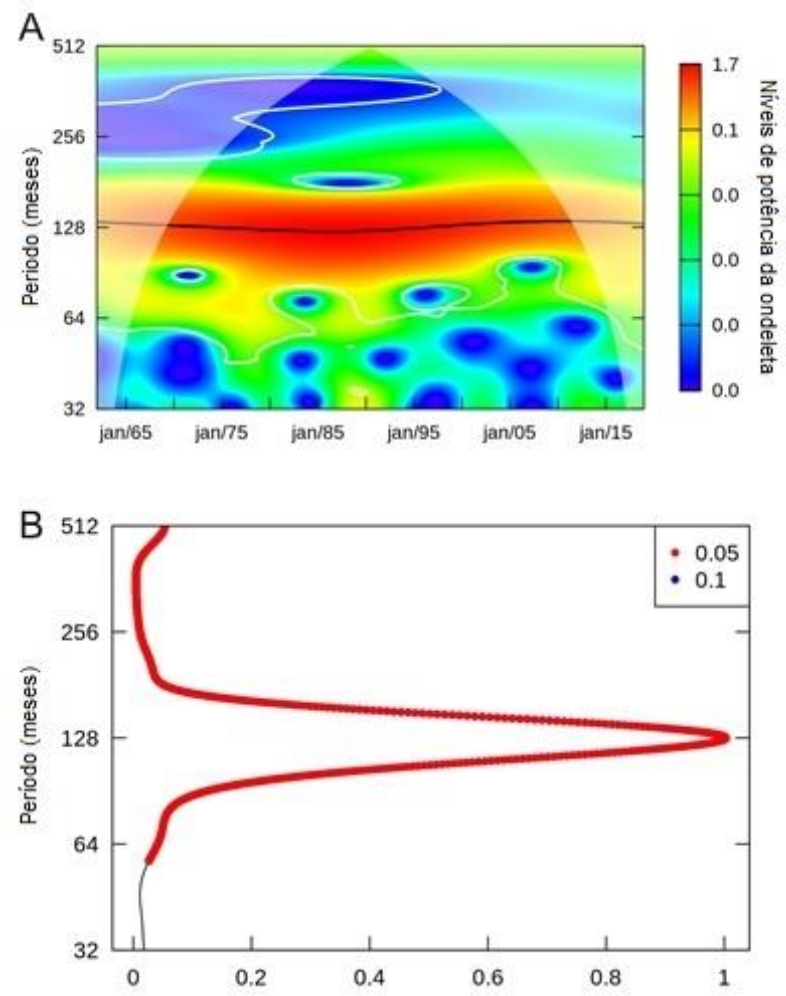

Figura 4. Análise de ondeleta para série de manchas solares. (A). Espectro de potência de ondeletas da série mensal de número de manchas solares, sendo o eixo $Y$ a escala (em meses), o eixo $X$ o tempo (em anos), e a escala de potência em cores. $O$ cone difuso delimita a região de influência e as linhas de contorno indicam períodos com $95 \%$ de significância. (B). Espectro integrado de ondeleta da série mensal de número de manchas solares, sendo o eixo Y o período (em meses); o eixo $X$ a média do espectro de potência; e as linhas em vermelho os períodos com 95\% de significância.

O espectro de ondeletas da série normalizada de anomalias de precipitação (Figura 5A) apresenta maior potência nos períodos correspondentes à altas frequências, em torno de 10 e 16 meses (Figura 5B) ao longo de todo o intervalo temporal analisado, com 95\% de significância estatística. Períodos de baixa frequência também foram identificados ( 250 meses ou 21 anos) (Figura 5B), a partir do final da década de 1970 (Figura $5 A)$.
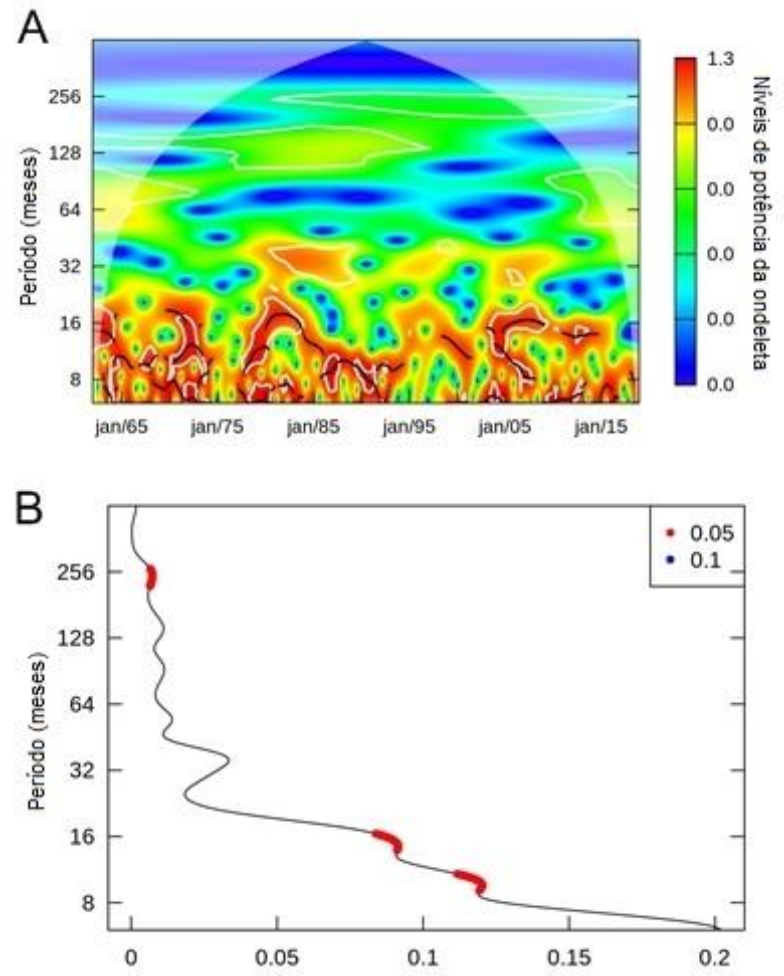

Figura 5. Análise de ondeleta para série de precipitação. (A) Espectro de potência de ondeletas da série mensal de anomalia de precipitação normalizada, sendo o eixo $Y$ a escala (em meses), o eixo $X$ o tempo (em anos), e a escala de potência em cores. $O$ cone difuso delimita a região de influência e as linhas de contorno indicam períodos com $95 \%$ de significância. (B). Espectro integrado de ondeleta da série mensal de anomalia de precipitação normalizada, sendo o eixo $Y$ o período (em meses); 0 eixo $X$ a média do espectro de potência; $e$ as linhas em vermelho os períodos com $95 \%$ de significância.

O espectro de ondeleta obtido para a série de número de manchas solares identificou pico espectral estatisticamente significativo de período de 128 meses ( 11 anos), ao longo de todo o intervalo de tempo. Esta ciclicidade está provavelmente associada ao ciclo de Schwabe de variação de manchas solares, com período de 11 anos (Echer et al, 2003). A menor potência espectral relacionada a frequências mais baixas estariam relacionadas ao ciclo de 22 anos de variabilidade solar, associado à variação do campo magnético solar (Echer et al, 2003).

No caso da variação de precipitação, análise semelhante à apresentada neste trabalho foi feita por Silva et al. (2009). Os autores aplicaram a transformada de ondeleta na série de precipitação da bacia do Mundaú, nordeste do Brasil, de 1955 a 1991. Foram identificados picos espectrais estatisticamente significativos em 20-22 anos e 4,6-5 anos, os quais foram atribuídos à influência da variabilidade de temperatura do Oceano Pacífico em 
escalas decadal e interanual, respectivamente. No presente trabalho, foram encontrados picos espectrais estatisticamente significativos de períodos 21 anos, além de 10 e 16 meses na variabilidade de precipitação da estação Brasília, no centro-oeste do Brasil. Estudos anteriores identificaram variabilidade decadal e interanual de precipitação no Sistema de Monção da América do Sul associada a variações de temperatura no Oceano Pacífico (e.g., Kayano et al., 2007). A Monção da América do Sul é o principal sistema associado ao regime de precipitação do Brasil Central (Gan et al., 2004). Assim, sugere-se investigação mais detalhada em relação a tais ciclicidades encontradas de forma a se verificar se o pico espectral de baixa frequência encontrado na série da estação Brasília corresponde a este sinal encontrado na Monção da América do Sul.

\section{Conclusão}

A aplicação do método da transformada de ondeletas como ferramenta estatística na análise de sinais geofísicos não-estacionários de origem terrestre (precipitação) e extraterrestre (número de manchas solares) se mostrou efetiva e robusta na detecção de picos espectrais significativos em diferentes escalas de tempo, pois as frequências encontradas corroboram com fenômenos identificados previamente na literatura. A aplicação da transformada de ondeletas apresentada neste trabalho corresponde a resultados preliminares para determinação da metodologia a ser utilizada em trabalhos futuros. Como etapas futuras, pretende-se investigar possíveis relações entre a variabilidade da atividade solar (representada pelas manchas solares) e o sinal climático em superfície do Brasil Central, dado pela estação meteorológica de Brasília.

\section{Referencias}

BOLZAN M. J. A. Análise da Transformada em Ondeletas Aplicada em Sinal Geofísico. Revista Brasileira de Ensino de Física, v. 26, n. 1, p. 37-41, 2004.

ECHER E., RIGOZO N. R., NORDEMANN D. J. R., VIEIRA L. E. A., PRESTES A., E FARIA H. H. O Número de Manchas Solares, Índice da Atividade do Sol. Revista Brasileira de Ensino de F'ısica, vol. 25 p. 157-163, 2003.

GAN M. A., KOUSKY V. E., E ROPELEWSKI C. F. The South America Monsoon Circulation and Its Relationship to Rainfall over West-Central Brazil. International Journal of Climatology, p. 47-63, 2004.

GÓMEZ J. M. R., CARLESSO F., VIEIRA L. E., E SILVA L. A Irradiância Solar: Conceitos Básicos. Revista Brasileira de Ensino de Física, vol. 40, nํㅜ, e3312, 2018.

KAYANO M. T. AND ANDREOLI R. V. Relations of South American Summer Rainfall interannual variations with the Pacific Decadal Oscillation. International Journal of Climatology, 27: p. 531-540, 2007.

SILVA D. F., SOUSA F. A. S., E KAYANO M. T. Uso de IAC E Ondeletas para Análise da Influência das Multiescalas Temporais na Precipitação da Bacia do Rio
Mundaú. Revista de Engenharia Ambiental, v. 6, n. 1, p. 180-195, 2009. 